\title{
Comparison - Effectiveness of oral steroid versus intratympanic Dexamethasone for sudden onset sensorineural hearing loss.
}

Rupasinghe R.A.S.T.

National Hospital of Sri Lanka.

\begin{abstract}
Background

Sudden sensory neural hearing loss is an ENT emergency, where $90 \%$ are idiopathic. Steroids are the mainstay of treatment, but the best mode of delivery need to be determined.
\end{abstract}

Aim - This study was carried out to determine the effectiveness of oral steroid versus intratympanic Dexamethasone for sudden onset sensory neural hearing loss

\section{Method}

Prospective, interventional, randomised, comparative study was carried out at the ENT unit at the National Hospital of Sri Lanka from September 2013 to August 2015.

\section{Results}

7 patients who met the inclusion criteria were randomly allocated in to two groups, and were treated with oral Prednisolone and Intratympanic Dexamethasone, which included 23 males and 14 females $(n=37)$. Within the present study, 85\% $(17 / 20)$ patients who had oral Prednisolone and $94.10 \%$ (16/17) patients who had intratympanic Dexamethasone clinically improved after treatment. Overall improvement following ITD is significantly higher $94.10 \%(\mathrm{P}<0.01)$. There were no adverse effects reported.

\section{Conclusion}

Intratympanic dexamethasone (ITD) is an effective treatment modality for patients presenting with sudden onset sensorineural hearing loss, compared to high dose oral steroids. Hearing improvement in low and mid frequencies are $100 \%$ compared to high frequencies. Early diagnosis and treatment, gave best results.

\section{Introduction.}

Sudden Sensorineural Hearing Loss (SSNHL)is a medical emergency and defined as sensory neural hearing loss of more than $10 \mathrm{~dB}$ in 3 consecutive frequencies within 3 days. These patients present with hearing impairment, tinnitus and ear fullness and also occasionally complain of dizziness and vertigo.

Diagnosis is by examination of the ears \& nose. Audiological examination includes a pure tone audiogram and a tympanogram. MRI scan of the brain preferably with gadolinium enhancement will exclude the presence of a CP angle tumour $[1,2]$.

The gold standard of treatment of SSNHL is high dose of steroids taken by mouth or intravenously. Injecting steroids into the middle ear is a relatively painless quick procedure done as an out-patient procedure. It is as effective as treating with high dose steroids but has less adverse effects than high dose oral or IV steroids.

\section{Literature Review}

Only 10 to $15 \%$ of people diagnosed with SSNHL have an identifiable cause. The most common causes are: Infectious diseases, Trauma such as a head injury, Autoimmune diseases such as Cogan's syndrome and Ototoxic drugs ${ }^{[3]}$.

Idiopathic sudden onset sensorineural hearing loss (ISSNHL) is a condition of unknown aetiology, affecting all age groups regardless of sex. So far in the literature there is evidence to confirm that steroid therapy is effective, but the best mode of administration is yet to be decided. Therefore, properly designed prospective, randomized, double blinded, controlled trials are required to determine the best mode of steroid therapy. Until 
then, we are of the opinion that patients should be offered a steroid therapy, provided there are no contraindications. Full explanation about possible side effect of steroid to the patients is essential.

In a non-randomized, retrospective review of patients presented with sudden sensorineural hearing loss of unknown cause, over a ten-year period with reported severe sensorineural hearing loss; steroid therapy significantly improved hearing recovery, compared to those who received no steroid therapy $(\mathrm{P}<0.01)$. The above effect was not found in patients with milder forms of hearing loss. Idiopathic sensorineural hearing loss mainly affects low frequencies, which is the normal speech frequency ${ }^{[4,5]}$

Idiopathic sudden sensorineural hearing loss has a high (50-70\%) spontaneous partial or complete recovery rate; therefore, for a given treatment to be considered effective, a very high success rate needs to be demonstrated. The studies shown are all small and offer no convincing evidence of recovery rates above those expected ${ }^{[6]}$

In another study a daily short-term intratympanic dexamethasone administration in ISSNHL patients without concurrent therapy showed a high response rate and high cure rate and proved to be an alternative therapeutic option to highdose systemic steroids, administered as a firstand/or second-line treatment ${ }^{[7]}$.

\section{Objectives}

General Objective

To assess and compare the effectivity of high dose oral steroids with intratympanic steroids.

Specific Objectives

\section{To assess the clinical improvement}

2. To assess patient satisfaction following the treatment modality

3. To compare the effectiveness between oral steroids and intra tympanic Dexamethasone treatment.

4. To assess and compare the adverse effects following the two treatment modalities.
5. To assess the effectiveness of treatment compared to duration of presentation.

6. To determine the most frequently affected frequencies in ISSNHL.

7. To determine the frequencies most commonly improved with steroid treatment.

\section{Material and Methods.}

This prospective, interventional, randomised, comparative study was carried out at the ENT unit at the National Hospital of Sri Lanka, from September 2013 to August 2015. Ethical clearance was obtained from the National Hospital Ethics Review Committee, and the consent and permission was obtained from the consultant ENT surgeons at the National Hospital to carry out the study. 53 patients were presented with SSNHL during the study period and 37 were included in the study according to the inclusion and exclusion criteria. Informed verbal consent was obtained from patients prior to the treatment and the procedure. Results and data were collected and recorded by the medical officers in respective units using pre prepared data sheets [annexure 1].

\section{Inclusion criteria}

1. Patients with a hearing loss of more than $10 \mathrm{~dB}$ in 3 consecutive frequencies

2. Patients presenting within 2 weeks of onset of hearing loss.

3. Patients with diabetes mellitus will be included with a referral to the resident physician regarding glycaemic control.

\section{Exclusion criteria}

1. Patients who have already been diagnosed and treated

\section{Patients on ototoxic medication}

37 patients who met the above criteria were randomly allocated in to two groups. Two separate registers were maintained in the male and female wards, and as the patients were admitted, they were alternatively allocated to the two treatment groups according to the register. Group 1 was 
given oral prednisolone and Group 2 was given intratympanic dexamethasone as follows.

1. Oral Prednisolone 60mg per day for 5 days

2.Intratympanic Dexamethasone $0.3-0.5 \mathrm{ml}$ stat and weekly up to three doses under local anaesthesia. Patients were given local anaesthesia using either $10 \%$ Xylocaine spray or EMLA cream according to patient's preference. The external canal was filled with 10\% xylocaine spray and kept for 15 minutes or an ear wick bathed in EMLA cream was kept over the tympanic membrane for 20 minutes before injection. A 29-gauge needle was used to administer the drug in to the middle ear. The procedure was carried out by a trained medical officer under microscopic guidance. Following injection, patients were kept lateral with the injected ear up for 20 minutes.

Pure tone Audiogram (PTA) was done in each of these patients of both groups on admission, at one week, one month and then at 3-month timeline, from the time of onset of treatment.

\section{Results.}

53 patients with SSNHL were presented to the unit during the study period. 37 patients who fulfilled the criteria were included in the study which included 23 males and14 females.

Age distribution of the study population categorized by gender is as follows (Figure 1):

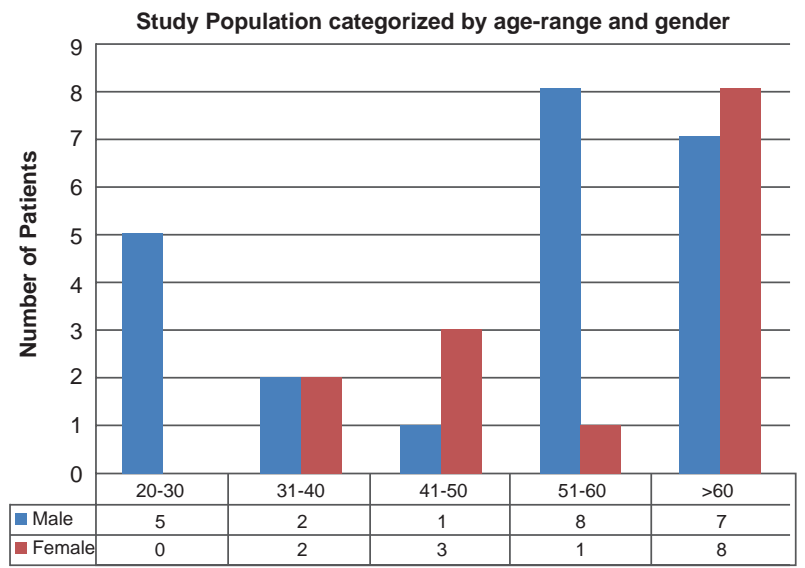

Figure 1: Study population by age and gender

All37 patients presented with hearing impairment. 21 had Right side hearing loss and 16 had left side hearing impairment. None of them presented with ear discharge or with a history of trauma. The results are as follows (Figure 2).

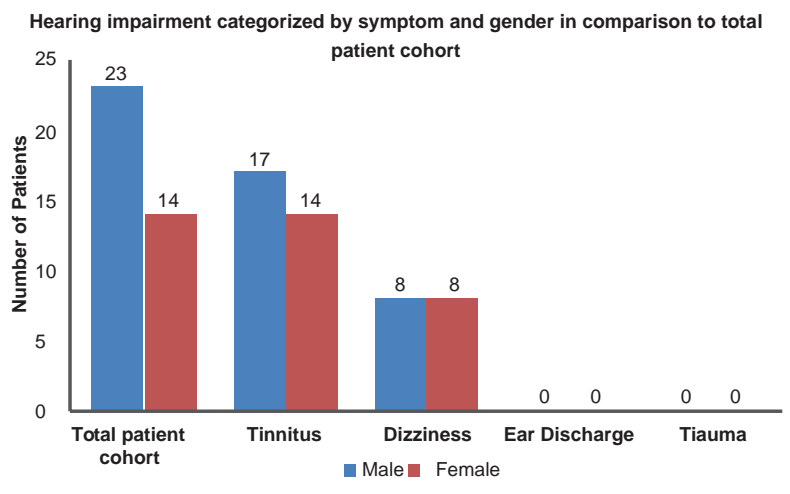

Figure 2: Hearing impairment categorized among male and female patients

The following co-morbidities were identified among the study population. And no adverse effects were noted following treatment (Table 1 and Figure 3)

Table 1: Patient medical history indicating comorbidities

\begin{tabular}{|l|c|c|c|c|}
\hline Medical history & male & $\begin{array}{c}\text { Per- } \\
\text { cent- } \\
\text { age }\end{array}$ & $\begin{array}{c}\text { fe- } \\
\text { male }\end{array}$ & $\begin{array}{c}\text { Per- } \\
\text { cent- } \\
\text { age }\end{array}$ \\
\hline Diabetes mellitus & 5 & $13 \%$ & 11 & $29 \%$ \\
\hline Hypertension & 9 & $24 \%$ & 7 & $18 \%$ \\
\hline $\begin{array}{l}\text { Ischemic heart } \\
\text { disease }\end{array}$ & 3 & $8 \%$ & 1 & $3 \%$ \\
\hline Bronchial asthma & 2 & $5 \%$ & 0 & $0 \%$ \\
\hline
\end{tabular}

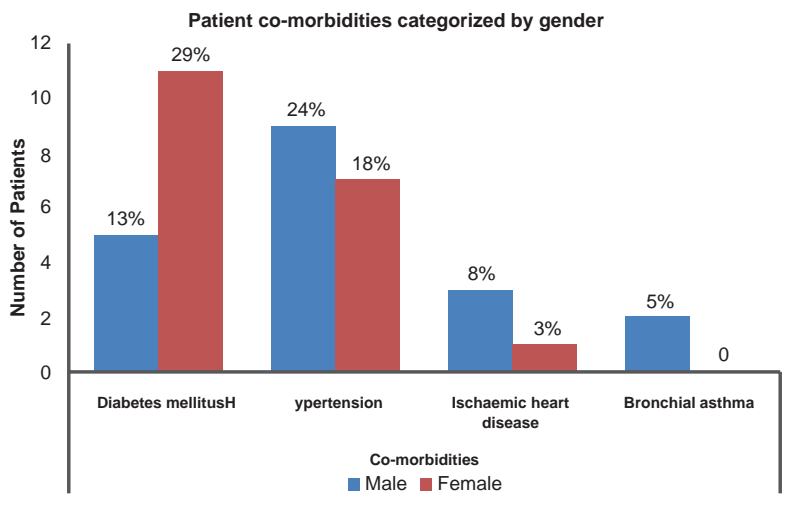

Figure 3: Patient co-morbidities categorized by gender

We found out that the patients were on long term medications which included: 
ISSN : $2012-855 x$

Ceylon Journal of Otolaryngology

(C) 2016; College of Otorhinolaryngologists and Head and Neck Surgeons of Sri Lanka

Metformin (2 patients $-5.40 \%)$, Daonil (1 patient-2.70\%), Lenteinsulin(9patients-24.32\%), Losartan (7 ptients-18.91\%), Atenolol (10 patients-27.02\%), Captopril (5 patients-13.51\%), ISMN (4 patients-10.81\%) and Serotide inhaler (2 patients-5.40\%)

\section{Examination findings}

Otoscopy revealed $100 \%$ negative findings with normal tympanic membranes and ear canals.

Tuning fork test revealed positive Rinnes test in 29 patients (78.37\%) and in 8 patients no air or bone conduction was seen in the affected ear (21. 62\%).Weber test showed lateralization to the opposite side in all patients, conforming SNHL on the affected side.

It was noted that the initial free field voice assessment has not been done in 20 patients (54.05\%).4 patients passed 6-inch whisper levels and 13 patients passed 6-inch conversation levels.

\section{Investigations}

Pure tone audiogram was done on admission, and subsequently at one week, one month and three months after treatment. Results were analysed as low, mid and high frequency levels on the affected side.

At presentation, 15 patients had high frequency loss, 12 patients had low frequency loss and 10 had mid frequency loss (Table 2 and Figure 4)

\begin{tabular}{|l|c|c|}
\hline Frequency range & $\begin{array}{c}\text { No of } \\
\text { patients }\end{array}$ & $\%$ \\
\hline Low $-125-500 \mathrm{~Hz}$ & 12 & $32.43 \%$ \\
\hline Mid $-500-2000 \mathrm{~Hz}$ & 10 & $27.02 \%$ \\
\hline High $-2000-8000 \mathrm{~Hz}$ & 15 & $40.54 \%$ \\
\hline
\end{tabular}

Table 2: Frequency range administered via pure tone audiogram

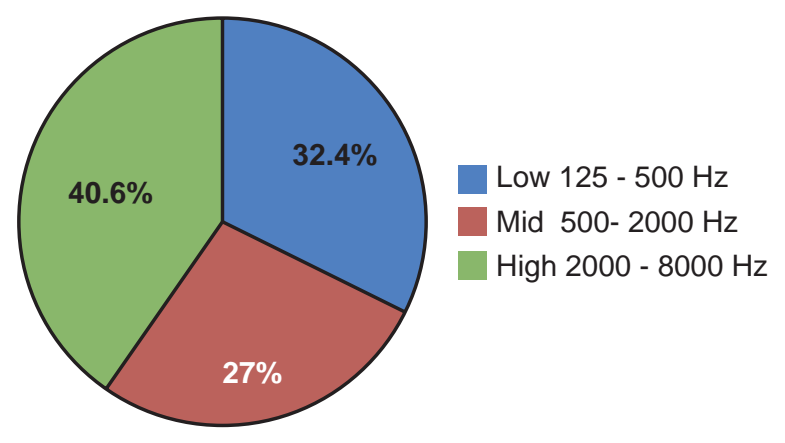

Figure 4: Percentage patient population in the low, mid and high frequency range.

Type A tympanogram was seen in all 37 patients (100\%).

Routine Full Blood Count, ESR. Fasting blood sugar. Total cholesterol, serum electrolytes and VDRL was done, but no significant abnormality was detected.

Treatment

20 patients who were included in group 1 had oral steroids (G1), and 17 patients in group 2 had intratympanic dexamethasone (G2).

In G1, 8 patients presented less than 3days of onset of symptoms, and 12 patients presented within 3 days to 2 weeks.

In G2, 11 patients presented less than 3 days of onset of symptoms, and 6 patients presented with in 3days to 2 weeks. (Table 3).

Table 3: Patient onset symptom presentation

\begin{tabular}{|l|c|c|c|c|}
\hline Presentation & G1 & $\%$ & G2 & $\%$ \\
\hline$<3$ days & 8 & $40 \%$ & 11 & $64.70 \%$ \\
\hline 3 days to $2 / 52$ & 12 & $60 \%$ & 6 & $35.29 \%$ \\
\hline
\end{tabular}

\section{Outcome- Group 1}

Out of 20 patients in group 1 who had oral steroids, 5 patients were completely improved after one week (25\%), 12 had mild improvement (5-10dB 
in 1-3 affected frequencies) (60\%) and 3 had no improvement after one week (15\%). One month follow up showed complete improvement in 6 patients (30\%), 11 patients were still in the mild range (55\%)and 3 had no improvement (15\%). In three months, 11 patients were lost to follow up (55\%).This included 6 who had complete improvement, 3who had no improvement and 2 who had mild improvement. 9 patients had the same results unchanged in the mild range (45\%) (Table 4 and Figure 5)

Table 4: Outcome tabulated over 3 months for group 1.

\begin{tabular}{|l|c|c|c|}
\hline Outcome & $1 / 52$ & $1 / 12$ & $3 / 12$ \\
\hline Complete improvement & 5 & 6 & lost \\
\hline Mild improvement & 12 & 11 & 9 \\
\hline No improvement & 3 & 3 & lost \\
\hline Lost to follow up & 0 & 0 & 11 \\
\hline
\end{tabular}

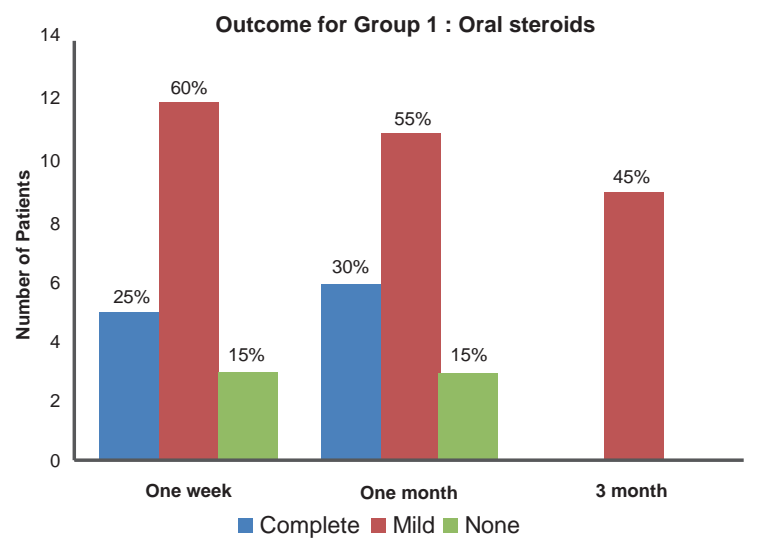

Figure 5: Outcome of 20 patients administered with oral steroids within one week, one month and 3 month follow up. At 3 months 55\% patients in the complete recovery and none recovery categories did not follow up.

Patient satisfaction - visual analogue scaleoral steroid group (G1)

In one week 5 patients were $100 \%$ satisfied with treatment, 12 patients were $50-60 \%$ satisfied with treatment and 3 patients were $20-30 \%$ satisfied with treatment. In one month, 6 patients were $100 \%$ satisfied with treatment. 11 patients were $50-60 \%$ satisfied with treatment and 3 patients were $20-30 \%$ satisfied with treatment. In three months, 11patients were lost to follow up and those who presented were only $50-60 \%$ satisfied with treatment.

\section{Outcome- Group 2}

Out of 17 patients in group 2 who had intra tympanic dexamethasone, 8 patients were completely improved after one week (47.05\%), 8 had mild improvement (5-10dB in 1-3 affected frequencies) and 1 had no improvement after one week $(5.88 \%)$. One month follow up showed complete improvement in 10 patients (58.82\%). 6 patients were still in the mild range (35.29\%)and 1 had no improvement (5.88\%). In three months, 2 patients were lost to follow up (11. 76\%).This included 1 who had complete improvement and 1 who had no improvement, 12 patients were completely improved $(70.58 \%)$ and 3 had the same results unchanged in the mild range (17.64\%) (Table 5 and Figure 6).

Table 5: Outcome tabulated over 3 months for group 2.

\begin{tabular}{|l|l|l|l|}
\hline Outcome & $1 / 52$ & $1 / 12$ & $3 / 12$ \\
\hline Complete improvement & 8 & 10 & 12 \\
\hline Mild improvement & 8 & 6 & 3 \\
\hline No improvement & 1 & 1 & lost \\
\hline Lost to follow up & 0 & 0 & 2 \\
\hline
\end{tabular}

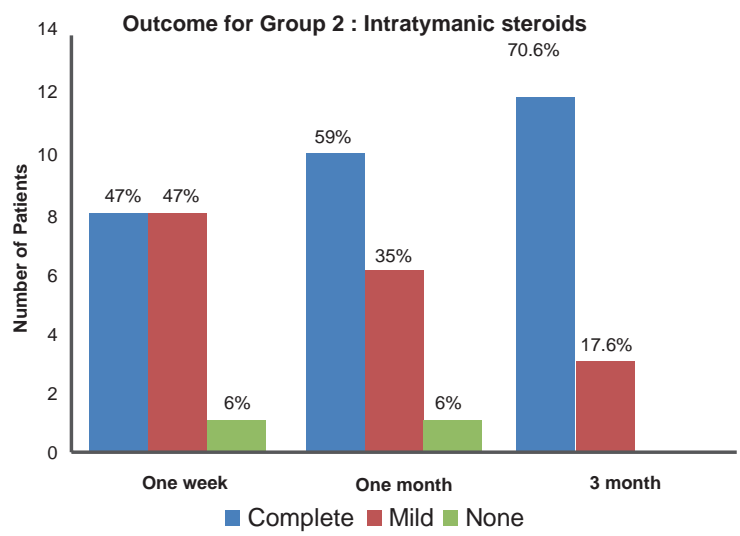

Figure 6: Outcome of 17 patients administered with intratympanic dexamethasone. At 3 months $11.8 \%$ patients in the complete improvement and no improvement categories did not follow up. 
ISSN : $2012-855 x$

Ceylon Journal of Otolaryngology

(C) 2016; College of Otorhinolaryngologists and Head and Neck Surgeons of Sri Lanka

Patient satisfaction - visual analogue scaleIntra tympanic dexamethasone group (G2)

In one week 8 patients were $100 \%$ satisfied with treatment. 8 patients were $50-60 \%$ satisfied with treatment and 1 patient was only $20-30 \%$ satisfied with treatment. In one month, 10 patients were $100 \%$ satisfied with treatment. 6 patients were $50-60 \%$ satisfied with treatment and 1 patient was only $10 \%$ satisfied with treatment. In three months, 2 patients were lost to follow up and 12 were $100 \%$ satisfied with the treatment. Whereas 3 were only $40 \%$ satisfied with treatment.

Adverse effects -no adverse effects were reported on either study groups during the study period. In this study the affected frequency ranges are detailed in table 5 .

\section{Table 6: Frequency ranges}

\begin{tabular}{|c|c|c|c|}
\hline Frequency range & $\begin{array}{c}\text { No of } \\
\text { patients } \\
\text { presented }\end{array}$ & $\begin{array}{c}\text { No of } \\
\text { patients } \\
\text { improved } \\
\text { complete- } \\
\text { ly }\end{array}$ & $\begin{array}{c}\text { No of } \\
\text { patients } \\
\text { improved } \\
\text { partially }\end{array}$ \\
\hline $\begin{array}{l}\text { Low - } \\
125-500 \mathrm{~Hz}\end{array}$ & $\begin{array}{c}12 \\
(32.43 \%)\end{array}$ & $\begin{array}{c}12 \\
(100 \%)\end{array}$ & \\
\hline $\begin{array}{l}\text { Mid - } \\
500-2000 \mathrm{~Hz}\end{array}$ & $\begin{array}{c}10 \\
\text { (27.02\%) } \\
\end{array}$ & $4(40.0 \%)$ & $6(60 \%)$ \\
\hline $\begin{array}{l}\text { High - } \\
2000-8000 \mathrm{~Hz}\end{array}$ & $\begin{array}{c}15 \\
(40.54 \%)\end{array}$ & $\begin{array}{c}2 \\
(13.33 \%)\end{array}$ & $9(60 \%)$ \\
\hline
\end{tabular}

\section{Discussion.}

In this study $85 \%(17 / 20)$ of patients who had oral Prednisolone, clinically improved after treatment. This includes 5 patients who had complete recovery (30\%) and 11 patients who had mild improvement after one month (55\%). Whereas $94.10 \%(16 / 17)$ of patients who had intratympanic Dexamethasone, clinically improved after treatment. This includes 13 patients who had complete recovery (76.47\%) and 3 patients who had mild improvement after three months (17.64\%).
$30 \%(6 / 20)$ of patients who had oral prednisolone were $100 \%$ satisfied with treatment and $45 \%$ $(9 / 20)$ were only $50-60 \%$ satisfied. $15 \%(3 / 20)$ was $20-30 \%$ satisfied $1 / 12$ after treatment. whereas $76.47 \%(12 / 17)$ patients who had IT dexamethasone were $100 \%$ satisfied with the treatment and $17.64 \%$ (3/17) were only 50-60\% satisfied with treatment.

Overall improvement of hearing following oral Prednisolone (both complete and partial recovery) after one month is $85 \%(17 / 20)$. Whereas overall improvement following ITD is $94.10 \%(16 / 17)$. The results are statistically significant $(\mathrm{P}<0.01)$ There were no adverse effects reported on either study groups during the study period.

Patients who presented $<3$ days showed 75 $\%$ recovery $(6 / 8)$ in Group 1 who had oral Prednisolone and100 \% recovery (11/11) was seen in Group 2 who had IT dexamethasone. The results are statistically significant. $(\mathrm{p}<0.01)$.

Out of the patients who presented within 3 days to $2 / 52,16.66 \%(2 / 12)$ patients in Group 1 who had oral steroids showed complete recovery and $75 \%(9 / 12)$ showed partial (mild) recovery. In Group 2who had IT dexamethasone $50.0 \%(3 / 6)$ showed complete recovery and $33.33 \%(2 / 6)$ showed partial (mild) recovery. The results are not statistically significant.

According to this study mid frequencies are the least affected, compared to the high and low frequencies

The overall improvement in hearing is seen in low and mid frequencies compared to high frequencies.

Intratympanic dexamethasone is an effective treatment modality for patients presenting with sudden onset sensorineural hearing loss, compared to high dose oral steroids. Hearing improvement in low and mid frequencies are $100 \%$ compared to high frequencies.

Earlier administration of treatment, gave best results. Sample size was less than expected. 
This is because in some instances without randomly allocating the patients, intra tympanic dexamethasone had been given especially in young age groups. Further assessment and study with a bigger sample and long term outcome after 3 months should be assessed to come to a definitive conclusion.

\section{Key Messages.}

Intratympanic dexamethasone (ITD) is an effective treatment modality for patients presenting with sudden onset sensorineural hearing loss, compared to high dose oral steroids. Hearing improvement in low and mid frequencies are $100 \%$ compared to high frequencies. Early diagnosis and treatment, gave best results.

\section{References.}

1.Weber, P.C., Zbar, R.I., Gantz, B.J.S.O., Application of high-dose orally applied dexamethasone seems to improve the recovery outcomes in comparison to prednisone 100 mg p.o. for 7 days.Otolaryngol Head Neck Surg. 1997;116(2):153

2.Egli, Gallo, D., Khojasteh, E., Gloor, M., Hegemann, S.C.A., Effectiveness of Systemic High-Dose Dexamethasone Therapy for Idiopathic Sudden Sensorineural Hearing Loss,AudiolNeurotol 2013;18:161-170 (DOI:10.1159/000346938)
3. Sudden sensory neural hearing loss, National institute on deafness and other communication disorders.(NIDCD) , US department of health and human services.

4.Chen, C.Y., Halpin, C., Rauch, S.D., Oral steroid treatment of sudden sensorineural hearing loss: a ten year retrospective analysis. OtolNeurotol. 2003;24(5):728-733. [PubMed]

5.Gheriani, H., Khan, M.H., Charles, D.A., Steroid Use in Idiopathic Sudden Sensorineural Hearing Loss: What is the Evidence? AudiolNeurotol 2006;11:331-338

6.Angaj Ghosh, Rupert Jackson,. Steroids in sudden sensorineural hearing loss; kevin. mackway-jones@man.ac.uk

7.Kakehata, S., Sasaki, A., Futai, K., Kitani, R., Shinkawa, H., Daily Short-Term Intratympanic Dexamethasone Treatment Alone as an Initial or Salvage Treatment for Idiopathic Sudden Sensorineural Hearing Loss. AudiolNeurotol2011;16:191-197 (DOI: 10.1159/000320210.1159/0003202 